\title{
Consumo habitual de alimentos fonte de vitamina A em pré-escolares da zona rural no Norte de Minas Gerais
}

\author{
Habitual consumption of vitamin a food sources \\ by preschool children in rural areas in \\ Northern Minas Gerais, Brazil
}

Paula Karoline Soares FARIAS ${ }^{1}$

Vanessa Santos SILVA ${ }^{1}$

Marise Fagundes SILVEIRA²

Antônio Prates CALDEIRA²

Lucinéia de $\mathrm{PINHO}^{1,2}$

\section{RE S U M O}

\section{Objetivo}

Avaliou-se o consumo habitual de alimentos fontes de vitamina A em pré-escolares da zona rural de Montes Claros, Minas Gerais.

\section{Métodos}

Em estudo do tipo transversal foram pesquisados 337 pré-escolares por meio de entrevista com seus pais. Foram avaliados: a) características socioeconômicas da família; b) recebimento de suplementação de vitamina A provida pelo governo, c) o hábito de consumo de alimentos fontes de vitamina A através de um Questionário de Frequência Alimentar. O hábito de consumo foi classificado entre alto/moderado ou baixo e associado às variáveis socioeconômicas usando-se o modelo de regressão de Poisson para calcular a razão de prevalência e respectivo intervalo de confiança a $95 \%$.

\section{Resultados}

A maioria das crianças era de famílias de baixa renda. Apenas $51 \%$ delas receberam pelo menos 4 doses de suplementação de vitamina A. O hábito de consumo de fontes dessa vitamina inferido pelo Questionário de Frequência Alimentar foi considerado alto/moderado para apenas 75 dos pré-escolares e baixo para 262 deles. $O$ baixo consumo de alimentos fontes de vitamina $A$ foi associado à renda $\leq 1$ salário mínimo $(R P=1,178)$ e consumo habitual de guloseimas ( $R P=1,256)$.

\footnotetext{
${ }^{1}$ Faculdade de Saúde Ibituruna, Curso de Nutrição, Departamento de Nutrição. Montes Claros, MG, Brasil.

2 Universidade Estadual de Montes Claros, Centro de Ciências Biológicas e da Saúde, Mestrado Profissional em Cuidado Primário. Campus Universitário Prof. Darcy Ribeiro, Vila Mauricéia, 39401-089, Montes Claros, MG, Brasil. Correspondência para/ Correspondence to: L PINHO. E-mail: <lucineiapinho@hotmail.com>.
} 


\section{Conclusão}

As crianças avaliadas apresentaram baixo registro de recebimento da suplementação de vitamina A provida pelo governo e baixo consumo de alimentos fonte desse nutriente, sendo este último associado à baixa renda familiar e ao alto consumo de guloseimas.

Palavras-chave: Consumo alimentar. Pré-escolar. Vitamina A.

\section{A B S T R A C T}

\section{Objective}

The present study evaluated the habitual consumption of vitamin A food sources in preschool children in rural areas of Montes Claros, Minas Gerais, Brazil.

\section{Methods}

This is a cross sectional study that includes 337 preschool children which were evaluated through an interview conducted with their parents. The parameters evaluated were: a) socioeconomic characteristics of the family; b) use of the vitamin A supplementation provided by the government, c) habitual consumption of vitamin A food sources, assessed using the Food Frequency Questionnaire. Vitamin A food source consumption was classified as high/moderate or low, and it was associated to the socioeconomic variables using the Poisson regression model to calculate the prevalence odds ratio and the respective 95\% confidence interval.

\section{Results}

Most children were from low income families. Only 51\% of them had received at least 4 doses of vitamin A supplementation. The habitual consumption of vitamin A sources, assessed using the food frequency questionnaire, was considered high/moderate for only 75 children and low for 262 children. The low of consumption of vitamin A sources was associated with living on minimum wage $(P R=1,178)$ and high consumption of sweets $(P R=1,256)$.

\section{Conclusion}

The data obtained indicate that the children evaluated lack vitamin A supplementation provided by the government and show low consumption of foods high in vitamin; the former was associated with low-income status and high consumption of sweets.

Keywords: Food consumption. Preschool children. Vitamin A.

\section{N T R O D U Ç Ã O}

A hipovitaminose A, ou Deficiência de Vitamina A (DVA), é considerada um grave problema de saúde pública, sendo uma das mais importantes deficiências nutricionais dos países em desenvolvimento ${ }^{1}$. Trata-se de uma condição que afeta aproximadamente 190 milhões de crianças pré-escolares (com menos de sete anos de idade) no mundo 2 e $17,4 \%$ das crianças brasileiras menores de cinco anos de idade ${ }^{3}$.

A deficiência de vitamina A primária, causada pelo consumo insuficiente da vitamina, comumente representando um estado de "fome oculta", manifesta-se de forma subclínica e sem indícios evidentes ${ }^{3}$. Uma vez que a vitamina $A$ participa de processos fisiológicos primordiais ao organismo, especialmente aqueles relacionados à visão, integridade tecidual e sistema imunológico, sua deficiência em pré-escolares pode prejudicar o aprendizado e o desenvolvimento ${ }^{2-4}$.

Para reduzir e controlar a DVA infantil o Ministério da Saúde brasileiro instituiu o Programa Nacional de Suplementação de Vitamina ${ }^{4}$, que a distribui gratuitamente nas Unidades Básicas de Saúde do Sistema Único de Saúde (SUS). A profilaxia da DVA é proposta a partir da suplementação de megadoses de vitamina $A$, ministradas semestralmente. O calendário ideal é de uma dose de $100.000 \mathrm{UI}$ (600 $\mu \mathrm{g}$ de retinol) para crianças de 6 a 11 meses e, dos 12 aos 59 meses, uma dose de $2000 \mathrm{UI}\left(1200 \mu \mathrm{g}\right.$ de retinol) a cada 6 meses $^{4}$. 
Embora a necessidade de vitamina A varie de acordo com a fase da vida e fatores como ingestão de lipídeos e exposição a doenças - parasitoses intestinais, diarreias decorrentes etc. -, a dose provida pelo programa de suplementação atinge a recomendada para crianças em idade pré-escolar-6. Porém, a abrangência do mesmo ainda é baixa, atingindo apenas $40 \%$ dos pré-escolares brasileiros ${ }^{3}$. Na análise dos dados regionalizados do Programa Nacional de Suplementação de Vitamina no ano de 2013, o estado de Minas Gerais apresentou cobertura de 51,21 e $6,04 \%$ das crianças de seis a 59 meses de idade para a primeira e segunda dose, respectivamente ${ }^{7}$.

$O$ incentivo do consumo de alimentos ricos em vitamina A é importante para prevenir a DVA 4 , especialmente considerando-se que o consumo desse nutriente por pré-escolares de diferentes regiões do País é inferior ao necessário ${ }^{8-11}$. Essa inadequação do consumo é condicionada por fatores culturais, tais como hábitos alimentares, preferências individuais e familiares e por fatores socioeconômicos que afetam a capacidade de escolha e compra desses alimentos. Assim, no intuito de contribuir para o conhecimento dos hábitos alimentares de brasileiros pré-escolares de diferentes regiões que possam levar à DVA, investigou-se, no presente estudo, o hábito de consumo de alimentos fontes de vitamina A por pré-escolares da zona rural de Montes Claros (MG).

\section{MÉ T O D O S}

Trata-se de um estudo transversal realizado com 337 pré-escolares com idade média de 48,3 $( \pm 11,4)$ meses e matriculados nas escolas da rede pública municipal na área rural da cidade de Montes Claros (MG), Brasil. A população urbana do município está estimada em 344427 habitantes e a rural em 17488 habitantes $^{12}$.

De acordo com o senso escolar de 2013, o município possui 15 escolas em zona rural que atendem 514 crianças com idade entre 24 e 59 meses $^{13}$. Para este estudo o procedimento de amostragem foi do tipo aleatório simples, a partir da lista de alunos enviada por cada escola. Estimou-se a participação de no mínimo 278 crianças, considerando os seguintes critérios para o cálculo amostral: número total de alunos, nível de confiança de $95 \%$, um erro amostral de $4 \%$ e a prevalência de deficiência de vitamina A de 50\%.

A coleta de dados foi realizada por duas acadêmicas de Nutrição devidamente treinadas e calibradas. Os dados das crianças foram fornecidos pelos responsáveis através de entrevistas realizadas no primeiro semestre de 2014 em horário de reuniões de pais.

As entrevistas foram pautadas em um formulário estruturado dividido em três seções, as quais compreendiam: descrição dos dados socioeconômicos, informações sobre o recebimento da suplementação de vitamina A suprida pelo governo e o Questionário de Frequência Alimentar (QFA) para detecção do hábito de consumo de fontes de vitamina $A$.

Para caracterização sociodemográfica e econômica da amostra, levantaram-se dados sobre idade e sexo da criança, escolaridade da mãe (dividido entre fundamental completo ou menos e ensino médio incompleto ou mais), atividade remunerada materna, número de moradores no domicílio (incluindo a criança), renda mensal da família, tipo de moradia, tipo de construção do domicílio, disponibilidade de água e fonte, esgotamento sanitário e coleta de lixo. O registro de recebimento de suplementação de vitamina $A$ provida pelo governo era confirmado pelos dados do cartão e/ou caderneta de saúde das crianças, cujos pais foram instruídos a apresentar na reunião escolar.

O Questionário de Frequência Alimentar utilizado para detecção do hábito de consumo de fontes de vitamina A foi o baseado na proposta do International Vitamin A Consultative Group (IVACG). Esse instrumento semiquantitativo é validado para o estudo de crianças e utilizado em pesquisas semelhantes à presente ${ }^{14}$. O QFA não é um medidor direto do consumo de determinado alimento, mas permite estimar o hábito de consu- 
mo (raro/inexistente, mensal, semanal e diário) de alimentos específicos, predizendo a relação dieta-doença. No presente estudo, o QFA avaliou o consumo de alimentos fonte de vitamina A que foram citados pelos entrevistados.

Estes foram classificados como fontes de alto, moderado ou baixo teor de vitamina $A$ de acordo com os níveis de retinol descritos pelo IVACG ${ }^{15}$. Tabelas específicas de Fontes de Carotenoides ${ }^{16}$ e Composição de Alimentos ${ }^{17}$ foram também usadas para classificação dos alimentos citados nas entrevistas e não listados pelo IVACG. Dessa forma, consideraram-se com alto teor de vitamina A aqueles com mais de 1.200 ug-ER; teor moderado aqueles com 100 a 1.199 ug-ER; e baixo teor aqueles com menos de 100 ug-ER. Considerando-se as particularidades de erros alimentares para a faixa etária estudada, registrou-se também o consumo de guloseimas (refrigerantes, biscoitos, doces e salgadinhos).

Os dados do questionário foram lançados em um banco de dados no programa Statistical Package for the Social Sciences (SPSS Inc., Chicago, llinois, Estados Unidos) e tratados através da estatística descritiva, com cálculo de frequências e percentuais. Para análise dos mesmos, os pré-escolares foram subdivididos em dois grupos: aqueles que consumiam alimentos que representavam fontes moderadas e altas de vitamina A diariamente e aqueles que não consumiam. Assim, a investigação dos fatores associados ao consumo habitual de fontes dessa vitamina foi feita comparando-se ambos os grupos.

A associação entre o consumo habitual de alimentos fontes de vitamina A entre os pré-escoalres e as variáveis explicativas foi investigada com base na Razão de Prevalência (RP), calculada pelo modelo de regressão de Poisson com variância robusta, e seu intervalo de confiança a 95\%. As associações significativas até o nível de $20 \%(p<0,02)$ na análise bruta foram incluídas num modelo de análise múltipla visando controlar possíveis fatores de confundimento. Adotou-se o nível crítico de $5 \%(p<0,05)$ para assumir as associações como estatisticamente significantes, no modelo final.

O estudo foi aprovado e apoiado pela Secretaria Municipal de Educação de Montes Claros e conduzido dentro dos preceitos éticos estabelecidos na Resolução n 466/2012 do Conselho Nacional de Saúde sobre pesquisas com seres humanos. O projeto de pesquisa foi aprovado pelo Comitê de Ética em Pesquisa sob o Parecer $n^{\circ} 530.598 / 14$. Por se tratar da avaliação de menores de idade, todos os responsáveis assinaram o Termo de Consentimento Livre e Esclarecido.

\section{RES U LTA D OS}

Das 337 crianças, 51,9\% eram do sexo feminino e $71,5 \%$ eram pardas. A Tabela 1 mostra os dados das variáveis sociodemográficas. Considerando os responsáveis respondentes, 83,4\% eram mães. Em relação à escolaridade, 59,3\% informou ter até o ensino fundamental completo ou incompleto e a maioria $(75,1 \%)$ vivia com um companheiro. Quanto à renda familiar, mais da metade dos respondentes possuía até um salário mínimo e era beneficiária do programa Bolsa Família. No tocante às condições de infraestrutura de moradia, observou-se que $57,6 \%$ das casas era provida de água tratada, porém, $77,4 \%$ não tinha acesso à rede de esgoto e 52,2\% à coleta pública de lixo. Quase todas as casas $(99,1 \%)$ tinham rede elétrica. Em relação à suplementação de vitamina A apenas cerca de metade das crianças $(51,0 \%)$ recebeu mais que quatro doses de suplementação.

Quanto ao consumo de fontes dessa vitamina, o único alimento de alto teor citado foi cenoura cozida. Os de teor moderado foram abóbora, acerola, cenoura crua, couve (folha), manga, margarina e ovo de galinha; já os de baixo teor foram abacate, alface, banana, caju, farinha de mandioca, goiaba, laranja, leite de vaca (em pó ou in natura), mandioca, mamão, melancia, milho, peixe e tomate. 
A análise do consumo de alimentos fontes de vitamina A (Tabela 2 ) revelou que $86,4 \%$ dos pré-escolares consumiam cenoura com frequência predominantemente semanal. Dentre os itens de teor moderado da vitamina, a cenoura crua e a couve foram os menos consumidos; em termos de consumo diário, a manga foi o mais ingerido e, no que diz respeito ao consumo semanal, o ovo de galinha e a abóbora foram os mais citados. Dentre os itens de baixo teor de vitamina A destacaram-se laranja, banana e leite. Em contrapartida, o consumo diário de balas e doces ofertados aos pré-escolares pelos familiares superou $45,0 \%$, e o consumo semanal de refrigerantes e salgadinhos industrializados foi maior que $85,0 \%$ (Tabela 3). Assim, a ingestão média de vitamina A diária calculada foi 349,29 $\mu$ g (de 30,60 $\mu \mathrm{g}$ a $765,59 \mu \mathrm{g}$ ) entre os pré-escolares e identificou-se que $78,9 \%(n=266)$ apresentaram indicativos de baixo hábito de consumo da mesma 5 .

Na Tabela 4 são apresentados os resultados da análise bivariada para testar associações entre consumo de alimentos fontes de vitamina $A$ e as características da população. O hábito de consumo dessas fontes, inferido pelo QFA, foi considerado alto/moderado para apenas 75 dos pré-escolares e baixo para 262 deles. As variáveis "renda" e "consumo de guloseimas" foram selecionadas para compor o modelo múltiplo para baixo consumo de alimentos fontes de vitamina A em pré-escolares. Na análise múltipla, as variáveis "baixa renda" e "consumo diário de guloseimas" se mantiveram associadas ao baixo hábito de consumo de fontes da vitamina pelos pré-escolares.

\section{I S C U S S Ã O}

O diagnóstico dietético por meio do uso do QFA tem sido utilizado como indicador de risco nutricional de populações, uma vez que as informações dietéticas fornecem subsídios para a identificação de comportamentos e hábitos alimentares. A interpretação dos dados obtidos por esse tipo de instrumento servem mais para indicar
Tabela 1. Caracterização sociodemográfica e suplementação de vitamina A de pré-escolares de zona rural $(n=337)$. Montes Claros (MG), 2014.

\begin{tabular}{|c|c|c|}
\hline Variáveis & $\mathrm{n}$ & $\%$ \\
\hline \multicolumn{3}{|l|}{ Sexo } \\
\hline Masculino & 162 & 48,1 \\
\hline Feminino & 175 & 51,9 \\
\hline \multicolumn{3}{|l|}{ Idade (meses) } \\
\hline 24 a 36 & 63 & 18,7 \\
\hline 37 a 48 & 103 & 30,6 \\
\hline$>48$ & 171 & 50,7 \\
\hline \multicolumn{3}{|l|}{ Cor da pele } \\
\hline Branca & 65 & 19,3 \\
\hline Não branca & 272 & 80,7 \\
\hline \multicolumn{3}{|l|}{ Estado civil (responsável) } \\
\hline Com companheiro & 253 & 75,1 \\
\hline Sem companheiro & 84 & 24,9 \\
\hline \multicolumn{3}{|l|}{ Escolaridade (responsável) } \\
\hline >Ensino médio incompleto & 137 & 40,7 \\
\hline SFundamental completo & 200 & 59,3 \\
\hline \multicolumn{3}{|l|}{ Renda familiar } \\
\hline >1 Salário mínimo & 104 & 30,9 \\
\hline s1 Salário mínimo & 233 & 69,1 \\
\hline \multicolumn{3}{|l|}{ Moradores da casa } \\
\hline 1 a 4 & 199 & 59,1 \\
\hline 5 a 6 & 138 & 40,9 \\
\hline$\geq 7$ & 55 & 16,3 \\
\hline \multicolumn{3}{|l|}{ Tipo de moradia } \\
\hline Própria & 244 & 72,4 \\
\hline Alugada & 32 & 9,5 \\
\hline Cedida & 61 & 18,1 \\
\hline \multicolumn{3}{|l|}{ Número de cômodos } \\
\hline 1 a 2 & 29 & 8,6 \\
\hline 3 a 4 & 109 & 32,3 \\
\hline 5 a mais & 199 & 59,1 \\
\hline \multicolumn{3}{|l|}{ Água tratada } \\
\hline Sim & 194 & 57,6 \\
\hline Não & 143 & 42,4 \\
\hline \multicolumn{3}{|l|}{ Rede de esgoto } \\
\hline Sim & 76 & 22,6 \\
\hline Não & 261 & 77,4 \\
\hline \multicolumn{3}{|l|}{ Coleta pública de lixo } \\
\hline Sim & 161 & 47,8 \\
\hline Não & 176 & 52,2 \\
\hline \multicolumn{3}{|l|}{ Energia elétrica } \\
\hline Sim & 334 & 99,1 \\
\hline Não & 3 & 0,9 \\
\hline \multicolumn{3}{|l|}{ Suplementação de vitamina $A$} \\
\hline Mais que 6 doses & 16 & 4,7 \\
\hline 4 a 5 doses & 156 & 46,3 \\
\hline 1 a 3 doses & 118 & 35,1 \\
\hline Nenhuma & 47 & 13,9 \\
\hline
\end{tabular}


Tabela 2. Frequência do consumo de alimentos com alto, moderado e baixo teor de vitamina A entre pré-escolares de zona rural. Montes Claros (MG), 2014.

\begin{tabular}{|c|c|c|c|c|c|c|c|c|c|c|}
\hline \multirow{3}{*}{ Teor de vitamina A } & \multicolumn{10}{|c|}{ Frequência de consumo } \\
\hline & \multicolumn{2}{|c|}{ Diário } & \multicolumn{2}{|c|}{ Semanal } & \multicolumn{2}{|c|}{ Mensal } & \multicolumn{2}{|c|}{ Raro/Nunca } & \multicolumn{2}{|c|}{ Total } \\
\hline & $\mathrm{n}$ & $\%$ & $n$ & $\%$ & $n$ & $\%$ & $n$ & $\%$ & $n$ & $\%$ \\
\hline \multicolumn{11}{|l|}{ Alto } \\
\hline Cenoura cozida & 75 & 22,3 & 122 & 36,2 & 94 & 27,9 & 46 & 13,6 & 291 & 86,4 \\
\hline \multicolumn{11}{|l|}{ Moderado } \\
\hline Cenoura crua & 70 & 20,8 & 58 & 17,2 & 38 & 11,3 & 171 & 50,7 & 166 & 49,7 \\
\hline Abóbora & 100 & 29,7 & 143 & 42,4 & 50 & 14,8 & 44 & 13,1 & 293 & 86,9 \\
\hline Couve & 77 & 22,8 & 89 & 26,4 & 50 & 14,2 & 121 & 35,9 & 216 & 64,1 \\
\hline Margarina & 109 & 32,3 & 116 & 34,4 & 35 & 10,4 & 77 & 22,9 & 260 & 77,1 \\
\hline Manga & 138 & 40,9 & 73 & 21,7 & 67 & 19,9 & 59 & 17,5 & 278 & 82,5 \\
\hline Acerola & 103 & 30,6 & 138 & 40,9 & 53 & 15,7 & 43 & 12,8 & 294 & 87,2 \\
\hline Ovo de galinha & 90 & 26,7 & 175 & 51,9 & 49 & 14,5 & 23 & 6,8 & 314 & 93,2 \\
\hline \multicolumn{11}{|l|}{ Baixo } \\
\hline Mamão & 91 & 27,0 & 88 & 26,1 & 62 & 18,4 & 96 & 28,5 & 241 & 71,5 \\
\hline Goiaba & 120 & 35,6 & 97 & 28,8 & 54 & 16,0 & 65 & 19,3 & 271 & 80,7 \\
\hline Peixe & 52 & 15,4 & 43 & 12,8 & 87 & 25,8 & 155 & 46,0 & 182 & 54,0 \\
\hline Tomate & 159 & 47,2 & 123 & 36,5 & 22 & 6,5 & 33 & 9,8 & 304 & 90,2 \\
\hline Abacate & 59 & 17,5 & 75 & 22,3 & 52 & 15,4 & 151 & 44,8 & 186 & 55,2 \\
\hline Mandioca & 97 & 28,8 & 121 & 35,9 & 50 & 14,8 & 69 & 20,5 & 268 & 79,5 \\
\hline Caju & 49 & 14,5 & 24 & 7,1 & 54 & 16,0 & 210 & 62,3 & 127 & 37,7 \\
\hline Melancia & 81 & 24,0 & 79 & 23,4 & 129 & 38,3 & 48 & 14,2 & 289 & 85,8 \\
\hline Leite de vaca & 237 & 70,3 & 67 & 19,9 & 15 & 4,5 & 18 & 5,3 & 319 & 94,7 \\
\hline Alface & 76 & 22,6 & 105 & 31,2 & 68 & 20,3 & 88 & 26,1 & 249 & 73,9 \\
\hline Milho & 108 & 32,0 & 56 & 16,6 & 75 & 22,3 & 98 & 29,1 & 239 & 70,9 \\
\hline Laranja & 156 & 46,3 & 118 & 35,0 & 44 & 13,1 & 19 & 5,6 & 318 & 94,4 \\
\hline Banana & 167 & 49,6 & 140 & 41,5 & 24 & 7,1 & 6 & 1,8 & 330 & 98,2 \\
\hline Farinha/mandioca & 108 & 32,0 & 107 & 31,8 & 55 & 16,3 & 67 & 19,9 & 270 & 80,1 \\
\hline
\end{tabular}

Tabela 3. Frequência do consumo de guloseimas entre os pré-escolares de zona rural. Montes Claros (MG), 2014.

\begin{tabular}{|c|c|c|c|c|c|c|c|c|c|}
\hline \multirow{3}{*}{ Alimentos } & \multicolumn{9}{|c|}{ Frequência de consumo } \\
\hline & \multicolumn{2}{|c|}{ Diário } & \multicolumn{2}{|c|}{ Semanal } & \multicolumn{2}{|c|}{ Mensal } & \multicolumn{2}{|c|}{ Raro/Nunca } & \multirow{2}{*}{ Total (\%) } \\
\hline & $n$ & $\%$ & & $\%$ & & $\%$ & $\mathrm{n}$ & $\%$ & \\
\hline Refrigerantes & 84 & 24,9 & 169 & 50,1 & 57 & 16,9 & 27 & 8,1 & 91,9 \\
\hline Salgadinhos & 84 & 24,9 & 139 & 41,2 & 70 & 20,8 & 44 & 13,1 & 86,9 \\
\hline Balas/doces & 153 & 45,4 & 104 & 30,9 & 52 & 15,4 & 28 & 8,3 & 91,7 \\
\hline Bolachas recheadas & 82 & 24,3 & 116 & 34,4 & 76 & 22,6 & 63 & 18,7 & 81,3 \\
\hline
\end{tabular}

um risco nutricional relacionado à carência ou ao excesso alimentar do que para diagnosticá-lo, pois o consumo é um indicador indireto do estado nutricional ${ }^{18}$. Além disso, as necessidades individuais de nutrientes podem se diferenciar substancialmente das recomendações diárias ${ }^{18}$. Apesar disso, a elevada prevalência de doenças associadas à dieta, presente tanto em países desen- volvidos como nos em desenvolvimento, justifica o investimento nesse tipo de pesquisa.

Com base no manual de suplementação de vitamina A do governo, as crianças da faixa etária estudada deveriam receber pelo menos uma dose da suplementação até os 12 meses e outras 8 doses até os 59 meses de idade ${ }^{4}$. Os participantes deste estudo tinham registros desa- 
Tabela 4. Análises bivariada e múltipla de associação entre as variáveis estudadas e o consumo habitual de alimentos fontes de vitamina A entre pré-escolares de zona rural. Montes Claros (MG), 2014.

\begin{tabular}{|c|c|c|c|c|c|c|}
\hline \multirow{3}{*}{ Variáveis } & \multicolumn{4}{|c|}{ Hábito de consumo } & \multirow{3}{*}{ RP (IC95\%) } & \multirow{3}{*}{$p$} \\
\hline & \multicolumn{2}{|c|}{ Alto e moderado } & \multicolumn{2}{|c|}{ Baixo } & & \\
\hline & $n$ & $\%$ & $n$ & $\%$ & & \\
\hline \multicolumn{7}{|c|}{ Análise bivariada } \\
\hline \multicolumn{7}{|l|}{ Sexo } \\
\hline Feminino & 38 & 21,7 & 137 & 78,3 & \multirow{2}{*}{$0,951(0,638-1,417)$} & \multirow{2}{*}{0,804} \\
\hline Masculino & 37 & 22,8 & 125 & 77,2 & & \\
\hline \multicolumn{7}{|l|}{ Idade } \\
\hline$>37$ meses & 58 & 21,2 & 216 & 78,8 & \multirow{2}{*}{$1,275(0,800-2,032)$} & \multirow{2}{*}{0,307} \\
\hline$\leq 36$ meses & 17 & 27,0 & 46 & 73,0 & & \\
\hline \multicolumn{7}{|l|}{ Cor da pele } \\
\hline Branca & 11 & 16,9 & 54 & 83,1 & \multirow{2}{*}{$1,390(0,779-2,482)$} & \multirow{2}{*}{0,265} \\
\hline Não branca & 64 & 23,5 & 208 & 76,5 & & \\
\hline \multicolumn{7}{|l|}{ Estado civil da mãe } \\
\hline Com companheiro & 56 & 22,1 & 197 & 77,9 & \multirow{2}{*}{$1,022(0,646-1,616)$} & \multirow{2}{*}{0,926} \\
\hline Sem companheiro & 19 & 22,6 & 65 & 77,4 & & \\
\hline \multicolumn{7}{|l|}{ Escolaridade (responsável) } \\
\hline >Ensino médio incompleto & 47 & 23,5 & 153 & 76,5 & \multirow{2}{*}{$1,003(0,887-1,135)$} & \multirow{2}{*}{0,958} \\
\hline sFundamental completo & 28 & 20,4 & 109 & 79,6 & & \\
\hline \multicolumn{7}{|l|}{ Renda familiar } \\
\hline >Salário mínimo & 32 & 42,6 & 72 & 57,3 & \multirow{2}{*}{$1,178(1,022-1,358)$} & \multirow{2}{*}{0,024} \\
\hline \Salário mínimo & 43 & 27,4 & 190 & 72,5 & & \\
\hline \multicolumn{7}{|l|}{ Guloseimas diariamente } \\
\hline Não & 44 & 58,6 & 110 & 41,4 & \multirow{2}{*}{$1,256(1,101-1,432)$} & \multirow{2}{*}{$<0,001$} \\
\hline $\operatorname{sim}$ & 31 & 42,0 & 152 & 58,0 & & \\
\hline \multicolumn{7}{|c|}{ Análise múltipla } \\
\hline Renda & & & & & $1,176(1,026-1,349)$ & 0,020 \\
\hline Consumo de guloseimas & & & & & $1,259(1,108-1,431)$ & $<0,001$ \\
\hline
\end{tabular}

Nota: RP: Razão de Prevalência; IC95\%: Intervalo de 95\% de Confiança.

tualizados e, embora uma boa parte tivesse a primeira dose documentada, a continuidade do tratamento não foi constatada, corroborando com outro estudo ${ }^{19}$. As crianças avaliadas tinham idade média de 48 meses e apenas $51 \%$ havia recebido mais que 4 doses da suplementação, quando deveriam ter recebido, em média, 7 doses.

A falha identificada no fornecimento de suplementação é preocupante, especialmente frente ao baixo consumo que a comunidade estudada faz de alimentos fonte de vitamina A. Esse resultado chama a atenção visto que a cobertura do programa de suplementação ainda é um desafio em algumas regiões do País ${ }^{7}$. Deve- -se considerar, ainda, que o estudo foi conduzido em zona rural, cujo acesso aos serviços de saúde pode ser difícil em razão da distância. Além disso, as áreas rurais brasileiras muitas vezes apresentam piores indicadores de renda, saneamento básico e níveis de escolaridade, o que os torna susceptíveis a DVA ${ }^{8,9}$. Esse resultado sugere que outros estudos sejam conduzidos na comparação de DVA entre as populações urbana e rural.

O Questionário de Frequência Alimentar mostrou a escolha de alimentos de alta disponibilidade na região, especialmente frutas como manga, tomate, laranja e banana. Desses, apenas a manga tem teor moderado de vitamina $\mathrm{A}$. O 
consumo de cenoura cozida, que seria a mais rica fonte de vitamina A dentre os itens listados, parece não ser suficiente para suprir a necessidade das crianças visto que praticamente $80 \%$ da população mostrou indicativos de baixo consumo desse nutriente. Deve-se ressaltar a associação da falta de hábito de alimentos fonte de vitamina A com o alto consumo de guloseimas. Considerando-se a faixa etária estudada, esse resultado é preocupante visto sugerir que alimentos saudáveis, fonte de vários nutrientes, são substituídos nas refeições por alimentos pobres em termos nutricionais. Esse padrão segue uma tendência mundial de aumento de consumo de alimentos ricos em lipídeos e açúcares por crianças ${ }^{20}$.

A carência de micronutrientes, em especial de vitamina $A$, pode resultar de práticas alimentares inadequadas, que por sua vez são comumente influenciadas por condições econômicas e culturais ${ }^{8-11,21}$. Isso foi confirmado no presente estudo, o qual mostrou que famílias de menor renda tinham piores hábitos de consumo de fontes desse nutriente. O quadro poderia ser atenuado com medidas simples como a inserção de alimentos regionais fontes da vitamina, de elevado valor nutritivo, baixo custo e fácil disponibilidade na merenda escolar ${ }^{22}$. Porém, como mostra uma pesquisa realizada na região metropolitana de Belo Horizonte (MG), esses alimentos ainda são pouco consumidos por pré-escolares por uma questão de falta de hábito ${ }^{23}$. Esses dados também foram evidenciados no presente estudo, onde as crianças avaliadas mostraram não consumir, de maneira frequente, alimentos fontes de carotenoides disponíveis na região. O consumo insuficiente de legumes e vegetais, padrão alimentar comum entre pré-escolares ${ }^{24,25}$, afeta o aporte de micronutrientes, em especial o de vitamina A, podendo influenciar negativamente na reação do sistema imunológico das crianças a infecções ${ }^{9,10}$. Programas de Educação Nutricional voltados à remodelação dos hábitos alimentares seriam uma alternativa para prevenção e controle da DVA $^{26}$.

\section{O N CLUS Ã O}

Conclui-se que as crianças avaliadas têm, além de falha no recebimento da suplementação de vitamina A provida pelo governo, baixo hábito de consumo de alimentos fonte desse nutriente. Os resultados sugerem que a ingestão de guloseimas e a baixa renda estejam associados ao baixo hábito de consumo de alimentos fonte de vitamina A. Sugere-se o desenvolvimento de um Programa de Educação Nutricional para o incentivo ao consumo desses alimentos na comunidade. Além de ser uma eficiente forma de prevenção de carências nutricionais, esse tipo de intervenção produz resultados positivos duradouros, uma vez que a formação de hábitos saudáveis desde a infância contribui para a promoção da saúde na vida adulta.

\section{COLABORADORES}

PKS FARIAS e VS SILVA participaram da concepção, do planejamento, da execução da pesquisa, da análise e interpretação dos dados e da elaboração e revisão do artigo. AP CALDEIRA colaborou na revisão crítica do artigo. MF SILVEIRA colaborou na análise dos dados e na revisão crítica do artigo. L PINHO participou da concepção, do planejamento e da orientação da pesquisa, da análise e interpretação dos dados e da elaboração e revisão do artigo.

\section{REFER Ê N C I A S}

1. Milagres RCRM, Nunes LC, Pinheiro-Sant'Ana HM. A deficiência de vitamina $A$ em crianças no Brasil e no mundo. Ciênc Saúde Colet. 2007; 12(5):1253-66. http://dx.doi.org/10.1590/S1413-8123200700 0500023

2. Organización Mundial de La Salud. Directriz: administración de suplementos de vitamina $A$ a lactantes y niños 6-59 meses de edad. Genebra: OMS; 2011.

3. Brasil. Ministério da Saúde. Pesquisa Nacional de Demografia e Saúde da Criança e da Mulher - PNDS 2006: dimensões do processo reprodutivo e da saúde da criança. Brasília: Ministério da Saúde; 2009 [acesso 2015 abr 25]. Disponível em: http:// bvsms.saude.gov.br/bvs/publicacoes/pnds_crianca_ mulher.pdf 
4. Brasil. Ministério da Saúde. Manual de condutas gerais do Programa Nacional de Suplementação de Vitamina A. 2 ${ }^{a}$ ed. Brasília: Ministério da Saúde; 2013 [acesso 2015 abr 25]. Disponível em: http:// bvsms.saude.gov.br/bvs/publicacoes/manual_ programa_nacional_vitamina_a_2edicao.pdf

5. Institute of Medicine. Dietary reference intakes: The essential guide to nutrient requirements. Washington (DC): The National Academies Press; 2006.

6. Padovani RM, Amaya-Farfan J, Colugnati FAB, Domene SMA. Dietary Reference Intakes: aplicabilidade das tabelas em estudos nutricionais. Rev Nutr. 2006; 19(6):741-60. http://dx.doi.org/ 10.1590/S1415-52732006000600010

7. Brasil. Ministério da Saúde. Programa Nacional de Suplementação de Vitamina A: divulgação dos resultados parciais do programa em 2013. Brasília: Ministério da Saúde; 2013 [acesso 2015 abr 25]. Disponível em: http://bvsms.saude.gov.br/bvs/ publicacoes/manual_condutas_suplementacao_ vitamina_a.pdf

8. Prado MS, Assis AMO, Martins MS, Nazaré MPA, Rezende IFB, Conceição MEP. Hipovitaminose A em crianças de áreas rurais do Semiárido Baiano. Rev Saúde Pública. 1995; 29(4):295-300. http://dx.doi. org/10.1590/S0034-89101995000400007

9. Santos MA, Rezende EG, Lamounier JA, Galvão MAM, Bonomo E, Leite RC. Hipovitaminose A em escolares da zona rural de Minas Gerais. Rev Nutr. 2005; 18(3):331-9. http://dx.doi.org/10.1590/S14 15-52732005000300005

10. Azevedo MMSA, Cabral PC, Diniz AS, Fisberg M, Fisberg RM, Arruda IKG. Deficiência de vitamina A em pré-escolares da cidade do Recife, Nordeste do Brasil. Arch Latinoam Nutr. 2010; 60(1):36-41.

11. Netto MP, Priore SE, Sant'Ana HMP, Peluzio MCG, Sabarense CM, Franceschini SCC. Fatores associados à concentração de retinol sérico em lactentes. Rev Paul Pediatr. 2012; 30(1):27-34. http://dx.doi.org/10.1590/S0103-058220120 00100005

12. Instituto Brasileiro de Geografia e Estatística. Censo demográfico 2010. Brasília: IBGE; 2010 [acesso 2014 jun 1]. Disponível em: http://www.censo 2010.ibge.gov.br/sinopse/index.php? uf=31\& dados $=29$

13. Qualidade Educacional. Censo escolar 2013: Montes Claros. [acesso 2014 ago 25]. Disponível em: http://www.qedu.org.br/cidade/2248-montesclaros/censo-escolar?year $=2013 \&$ dependence $=$ o\&localization=0\&item =

14. Aquino JS, Pessoa DCNP, Oliveira CEV, Cavalheiro JMO, Stamford TLM. Processamento de biscoitos adicionados de óleo de buriti (Mauritia flexuosa
L.): uma alternativa para o consumo de alimentos fontes de vitamina A na merenda escolar. Rev Nutr. 2012; 25(6):765-74. http://dx.doi.org/10.1590/S1 415-52732012000600008

15. International Vitamin A Consultive Group. Guidelines for the development of a simplified dietary assessment to identify groups at risk for inadequate intake of vitamin A: Report of the international vitamin A Consultative Group. Washington (DC): The Nutrition Foundation; 1989.

16. Rodrigues-Amaya DB, Kimura M, Amaya-Farfan J. Fontes brasileiras de carotenoides: tabela brasileira de composição de carotenóides em alimentos. Brasília: Ministério do Meio Ambiente; 2008.

17. Philippi ST. Tabela de composição de alimentos: suporte para decisão nutricional. $3^{a}$ ed. Barueri: Manole; 2012.

18. Falcão-Gomes RC, Coelho AAS, Schmitz BAS. Caracterização dos estudos de avaliação do consumo alimentar de pré-escolares. Rev Nutr. 2006; 19(6):713-27. http://dx.doi.org/10.1590/S1415-5 2732006000600008

19. Paiva AA, Cagliari MPP, Queiroz D, Soutto RA, Brito VRS, França ISX. Programa Nacional de suplementação de vitamina A no estado da Paraíba: uma análise a partir do relato de profissionais da Equipe de Saúde da Família. Epidemiol Serv Saúde. 2011; 20(3):373-83. http://dx.doi.org/10.5123/\$1679-4 9742011000300012

20. Alves MN, Muniz LC, Vieira MFA. Consumo alimentar entre crianças brasileiras de dois a cinco anos de idade: Pesquisa Nacional de Demografia e Saúde (PNDS), 2006. Ciênc Saúde Colet. 2013; 18(11):3369-77. http://dx.doi.org/10.1590/S1413-8 1232013001100026

21. Queiroz R, Paiva AA, Pedraza DF, Cunha MAL, Esteves GH, Luna JG, et al. Deficiência de vitamina A e fatores associados em crianças de áreas urbanas. Rev Saúde Pública. 2013; 47(2):248-56. http://dx. doi.org/10.1590/rsp.v47i2.76627

22. Silveira ML, Martins MC, Oliveira EKF, Joventino ES, Ximenes LB. Conhecimento, atitude e prática sobre alimentos regionais entre famílias de pré-escolares. Rev Rene. 2014; 15(1):37-44.

23. Barros ALA, Soares ADN, Pessoas MC, Teixeira RA, Beinner MA. Deficiência de vitamina a em crianças residentes na região metropolitana de Belo Horizonte, Minas Gerais. Rev Min Enferm. 2010; 14(3):386-93.

24. Nobre LN, Lamounier JA, Franceschini SCC. Padrão alimentar de pré-escolares e fatores associados. J Pediatr. 2012; 88(2):129-36. http://dx.doi.org/ 10.2223/JPED.2169 
25. Souza RLV, Madruga SW, Gigante DP, Santos IS, Barros AJD, Assunção MCF. Padrões alimentares e fatores associados entre crianças de um a seis anos de um município do Sul do Brasil. Cad Saúde Pública. 2013; 29(12):2416-26. http://dx.doi.org/10.15 90/0102-311X00156412

26. Gondim SSR, Diniz AS, Cagliari MPP, Araújo ES, Queiroz D, Paiva AA. Relação entre níveis de he- moglobina, concentração de retinol sérico e estado nutricional em crianças de 6 a 59 meses do Estado da Paraíba. Rev Nutr. 2012; 25(4):441-9. http:// dx.doi.org/10.1590/S1415-52732012000400002

Recebido: outubro 30, 2014 Versão final: maio 15, 2015 Aprovado: junho 10, 2015 